\title{
Teknokultura
}

ISSN: 1549-2230

\section{El programa Academia 3.0 y la evaluación del profesorado en el área de Sociología a partir de los índices de impacto de las revistas}

\author{
María José Rubio Martín; Pilar Parra Contreras²
}

Recibido: 22 de abril de 2017 / Aceptado: 9 de junio de 2017

Resumen. Este artículo analiza las principales diferencias entre el programa Academia 2.0 y el Academia 3.0, tras su publicación en noviembre de 2016, tomando como referencia la categoría de profesor titular en el ámbito de la investigación del área de Sociología. Uno de los aspectos destacados es el protagonismo que adquiere la actividad investigadora y la incorporación, como criterio obligatorio, de artículos publicados en revistas indexadas en los primeros cuartiles del Journal Citation Reports para la evaluación de las figuras de titular y catedrático de Universidad. El objetivo es mostrar las implicaciones, tanto académicas como políticas, que tienen estos cambios. Para ello, se realiza un análisis en el que se pone en relación las nuevas exigencias para la acreditación del profesorado con un contexto más amplio. Por un lado, el origen y alcance de los organismos de evaluación y el sistema de indización de las revistas académicas. Por otro, su conexión con lógicas de la cuantificación, la devaluación de la función docente, y el carácter neoliberal que tienen estas reformas en el ámbito universitario.

Palabras clave: escritura académica, revistas internacionales, investigadores españoles, evaluación científica, comunicación científica.

\section{[en] The Academia 3.0 program and the evaluation of the teaching staff in Sociology from the impact indexes of the journals}

\begin{abstract}
This article analyzes the main differences between the Academia 2.0 and Academia 3.0 programs, after its publication in November 2016. We take the category of senior lecturer in the field of research in Sociology as a reference point. One of the highlights is the centrality acquired by the research activity and the incorporation, as a mandatory criterion, of articles published in journals indexed in the first quartiles the Journal Citation Reports for the evaluation of the category of senior lecturer and university professors. The aim is to show the academic and policy implications of these changes. For this, an analysis is carried out in which the new requirements for the accreditation of teachers with a broader context are related. On the one hand, in this context, the origin and scope of the evaluation agencies and the indexing system of academic journals are considered. On the other, their connection with quantification logics, the devaluation of the teaching function, and the neoliberal character of these reforms in the university context are also given consideration.
\end{abstract}

Keywords: academic writing, international journals, spanish researchers, scientific evaluation, scientific community.

$1 \quad$ Universidad Complutense de Madrid

Email:mj.rubio@cps.ucm.es

2 Universidad Complutense de Madrid

E-mail: pparra@cps.ucm.es 
Sumario. 1. Introducción. 2. La evaluación del profesorado en el programa Academia 2.0 y Academia 3.0. 3. Journal Citation Report (JCR) y SCImago Journal Rank (SJR). 4. Más allá de la evaluación del profesorado. 5. Cierre. 6. Referencias bibliográficas

Cómo citar: Rubio Martín, M.J., Parra Contreras,P. (2017) El programa Academia 3.0 y la evaluación del profesorado en el área de Sociología a partir de los índices de impacto de las revistas, en Teknokultura 14, 105-119.

\section{Introducción}

La evaluación científica y académica se concreta a través de las agencias de evaluación, como institución básica que da o quita valor y reconocimiento a la labor de docentes e investigadores, tanto en el sistema general de la ciencia, como en el ámbito específico de la educación superior. La creación de estas agencias a partir de la finalización de la Segunda Guerra Mundial (cuando se crean muchas universidades, pero también cuando el declive del bloque soviético da paso a la expansión mundial del capitalismo económico financiero), supuso el establecimiento de una institución burocrática encargada de repartir reconocimiento y prestigio, reparto de recursos económicos y humanos, ordenación y estratificación de la comunidad científica. De esa manera, dicha comunidad se ha ido amoldando al control burocrático de las agencias evaluadoras que, a modo de "objetos frontera", se encargan de aprobar proyectos de investigación, evaluar actividades investigadoras, subvencionar proyectos, o avalar distintos niveles académicos (Fernández Esquinas et al., 2009).

Como es sabido, en su expresión última, ese control burocrático se articula a través de una serie de protocolos e indicadores (acreditaciones, estándares de calidad, número de publicaciones, publicaciones en revistas indexadas en determinadas bases de datos, sexenios de investigación, etc.), que abren o cierran las posibilidades de consolidar una carrera académica, realizar investigaciones, y participar en determinadas redes y equipos de investigación.

En el Estado español, ese conjunto de normas y procedimientos fue adoptado a finales del franquismo y durante la Transición democrática, con el fin de sustituir el viejo sistema de financiación discrecional por otro asentado en el principio de separación de niveles (político-organizativo-ejecutivo) de la ciencia. En la actualidad, la Ley 14/2011, de 1 de junio, de la Ciencia, la Tecnología y la Innovación, supone la pieza normativa clave del sistema de $\mathrm{I}+\mathrm{D}+\mathrm{i}$.

Este sistema cuenta con diferentes organismos reguladores entre niveles. En primer lugar, el Ministerio de Economía, Industria y Competitividad (MINECO), así como los diferentes organismos encargados en las Comunidades Autónomas, que representan las principales organizaciones de planificación política ${ }^{3}$. En segundo lugar, se encuentran la Agencia Estatal de Investigación (AEI), la Comisión Nacional Evaluadora de la Actividad Investigadora (CNEAI), y la Agencia Nacio-

3 La Ley 14/2011, de junio, de la Ciencia, la Tecnología y la Innovación ideó una Agencia Estatal de Investigación (AEI), que no fue creada hasta 2015 (Real Decreto 1067/2015, de 27 de noviembre). Sin embargo, y debido a los avatares políticos acecidos durante 2016, habrá que esperar a que el Real Decreto 1/2017, de 13 de enero, modifique el Real Decreto de 2015, cree definitivamente la AEI y apruebe su Estatuto. En esta nueva composición, la Agencia Nacional de Evaluación y Prospectiva (ANEP) queda integrada en la AEI. 
nal de Evaluación y Acreditación de la Calidad (ANECA), así como las agencias autonómicas de evaluación ${ }^{4}$. Por su parte, las universidades y las Organizaciones Públicas de Investigación (OPIs) constituyen los principales productores del conocimiento (nivel ejecutivo de la ciencia). Y finalmente, las empresas, aunque en mucha menor medida que las anteriores, representan otra fuente de conocimiento científico y tecnológico.

En todo el periodo democrático, este sistema de $\mathrm{I}+\mathrm{D}+\mathrm{i}$ se ha visto seriamente subordinado a los vaivenes económicos de cada época. Siempre escasamente dotado, el sistema científico español ha sido sensiblemente debilitado a partir de la crisis económico-financiera iniciada en 2008. Así, por ejemplo, mientras que en el periodo 2002-2009 el porcentaje de gasto del PIB en el sistema de I+D fue aumentando del $0,96 \%$ hasta el $1,35 \%$, a partir de entonces comenzó a decrecer hasta descender hasta valores de 2006 (0,69\%) (Fernández Esquinas et al., 2009; Fernández-Zubieta, 2014).

Pero, teniendo como telón de fondo este rasgo de infradotación económica, que condiciona toda la actividad científica española, nos interesa profundizar en otra característica del sistema que, a su vez, supone el objeto central de este artículo. A saber: el papel central que en el mismo cumplen las agencias evaluadoras que se acaban de mencionar. Para ello, en las siguientes páginas se revisan algunas de las principales diferencias entre el antiguo y el nuevo procedimiento de evaluación de los profesores titulares de Universidad por parte del programa Academia, después de su modificación en noviembre de 2016, tomando como referencia el ámbito de la investigación del área de Sociología. El objetivo último consiste en mostrar tanto las implicaciones académicas como las políticas que tienen estos cambios. Para ello, el análisis se pone en relación con un contexto más amplio. Por un lado, el origen y alcance de los organismos de evaluación y el sistema de indización de las revistas académicas. Por otro, su conexión con lógicas de la cuantificación, la devaluación de la función docente, y el carácter neoliberal que tienen estas reformas en el ámbito universitario.

\section{La evaluación del profesorado en el programa Academia 2.0 y Academia 3.0}

En el ámbito de la educación superior la Ley Orgánica de Universidades -LOU(2001), creada en el marco de la declaración de Bolonia (1999), supuso el inicio de la reforma de las enseñanzas y títulos oficiales, así como de la carrera académica, a cargo de la ANECA. Todo ello en un marco de adecuación a las directrices del Espacio Europeo de Educación Superior (EEES). La llamada "cultura de la evaluación", así como el "fomento de la excelencia” fueron los principios de primer orden invocados para la creación de esta agencia. Por su parte, el CNEAI, creado en 1989, evalúa la actividad investigadora de los profesores universitarios, con el objeto del reconocimiento de un complemento de productividad (sexenio). Finalmente, la ANEP (ahora integrada en la Agencia Estatal de Investigación -AEI-) se

4 Otras agencias de este tipo son las Oficinas de Transferencia de Resultados (OTRI), el Centro de Desarrollo Tecnológico e Industrial (CDTI), etc. 
encarga de examinar las propuestas de financiación de los proyectos de investigación.

La selección del profesorado se ha realizado durante las últimas décadas en función de tres sistemas diferentes. La ley Orgánica de Reforma Universitaria (LRU), de 1983, durante el Gobierno de Felipe González, la Ley Orgánica de Universidades (LOU) de 2001, de José Ma Aznar, y la ley orgánica de José L. Rodríguez Zapatero, de 2007, conocida como LOMLOU por modificar la LOU. La Agencia Nacional de Evaluación de la Calidad y Acreditación (ANECA) fue creada como Fundación en 2002 en el contexto de la Ley Orgánica de Universidades (LOU), como se ha señalado anteriormente, pero la LOMLOU potenció su papel al asignarle la realización de las acreditaciones (De Azcárraga, 2014), requisito imprescindible para poder optar a las plazas de profesores catedráticos y titulares que convocan las universidades.

Actualmente ANECA es un organismo autónomo adscrito al Ministerio de Educación, Cultura y Deporte, creado por el artículo 8 de la Ley 15/2014, de 16 de septiembre, procedente de la conversión de la Fundación Agencia Nacional de Evaluación de la Calidad y Acreditación en organismo público que tiene como objetivo contribuir a la mejora de la calidad del sistema de educación superior mediante la evaluación, certificación y acreditación de enseñanzas, profesorado e instituciones.

Tras su creación en 2002 se establecieron los criterios de evaluación de calidad del profesorado. Para la figura de titular de Universidad, el Real Decreto 1312/2007 estableció que la acreditación se realizaría por comisiones de acreditación y que los miembros fueran designados por el Consejo de Universidades (entre los propuestos por ANECA). Para esta figura se establecieron cuatro grandes apartados de criterios de evaluación: Actividad investigadora, actividad docente o profesional, formación académica y experiencia en gestión y administración.

La experiencia investigadora se configuraba en cinco bloques más un apartado adicional de otros méritos, y se valoraba hasta un máximo de 50 puntos sobre 100, con una especial atención a las publicaciones científicas en revistas, teniendo en cuenta las publicadas en revistas indexadas. En este punto se señalaba que las revistas podían estar en catálogos que asignan a cada revista un índice de calidad relativo, como son las publicaciones en revistas de reconocido prestigio e incluidas en los catálogos tipo Journal Citation Reports o equivalentes en cada especialidad, y publicaciones científicas no indexadas o sin índice de calidad relativo ${ }^{5}$.

En el campo de las Ciencias Sociales se valoraban preferentemente las publicaciones científicas en revistas de prestigio incluidas en listados tales como Science Citation Index, Social Sciences Citation Index, Econlit, catálogo de Latindex u otros listados generalmente admitidos en este campo. Y para obtener la puntuación máxima en este apartado en las áreas de Ciencias Económicas y Empresariales, Ciencias de la Educación, Ciencias de la Comunicación y Periodismo, Sociología, Ciencias Políticas y Ciencias de la Administración se consideraba necesario (como estándar) la publicación de un mínimo de 8 artículos en revistas recogidas en los

5 El programa Academia 2.0 ANECA tiene en cuenta la diversidad de ámbitos científicos. Además de considerar preferentemente las aportaciones que sean artículos en revistas de reconocido prestigio, también incluye otras bases de datos en función de las áreas de conocimiento (véase tabla 2). 
índices mencionados anteriormente, de las cuales un porcentaje significativo debían corresponder a revistas indexadas con un índice de calidad relativa. Este programa, denominado Academia 2.0, dejó de admitir solicitudes para la acreditación para profesores titulares y catedráticos de Universidad el 31 de diciembre de 2015.

Después de casi un año de espera, en noviembre de 2016 ANECA publicó algunos criterios del nuevo programa Academia 3.0. Durante ese tiempo tan solo se dio a conocer el Real Decreto 415/2015, de 29 de mayo, que modificaba el Real Decreto $1312 / 2007$, de 5 de octubre, y establecía los cambios normativos y los principales criterios del sistema de acreditación nacional para acceder a las figuras de profesor titular y catedrático de Universidad.

La publicación de estos documentos ha generado un notable rechazo por una parte de la comunidad académica. Además de poner de manifiesto la publicación incompleta de los criterios, lo que se destaca desde distintos ámbitos es la elevación del nivel de exigencia en muchos campos científicos, principalmente en el apartado de investigación, relacionado con el criterio obligatorio de tener artículos publicados en revistas indexadas en los Q1 y Q2 del Journal Citation Reports ${ }^{6}$.

Algunos de los principales cambios que se introducen en el RD 415/2015 se concretan en obtener la acreditación por rama de conocimiento (Artes y Humanidades, Ciencias, Ciencias de la Salud, Ciencias Sociales y Jurídicas, Ingeniería y Arquitectura), frente a la acreditación universal anterior. También se modifica el número de las comisiones de acreditación, que pasan de las 5 anteriores a 21 (más 5 comisiones de revisión), con el objetivo de que sean más especializadas y, se ha pasado de un sistema por puntos a un sistema alfabético. Las combinaciones posibles para obtener la calificación mínima para la acreditación según el sistema actual se resumen en la tabla 1 , y son las siguientes:

Tabla 1. Titular de Universidad programa Academia 3.0

\begin{tabular}{|l|c|c|c|c|c|}
\hline & Investigación & Docencia & $\begin{array}{c}\text { Transferencia/ } \\
\text { Actividad } \\
\text { profesional }\end{array}$ & Gestión & Formación \\
\hline Calificación mínima & B & B & & & \\
\hline Calificación mínima & A & C,E & & & B \\
\hline Calificación mínima & B & C & B & & B \\
\hline Calificación mínima & B & C & & B & B \\
\hline Calificación mínima & C & B & A & & B \\
\hline
\end{tabular}

6 Ante las numerosas peticiones realizadas por parte de la comunidad universitaria, la Conferencia de Rectores de las Universidades Españolas (CRUE) comunicó el día 19 de diciembre de 2016 en una nota de prensa en su página web, (www.crue.org), que había solicitado al Ministerio de Educación, Cultura y Deporte (MECD) analizar conjuntamente los citados criterios, con el fin de evaluar su impacto y arbitrar, si se estima oportuno, medidas tendentes a compensar cualquier posible efecto negativo que se pudiera derivar del cambio de normativa. A estas voces se sumaban, además, las de las organizaciones sindicales con representación en la Mesa Sectorial al considerar que los nuevos criterios son una exigencia desmedida tanto para el acceso a plazas estables de funcionario docente como para la promoción profesional de los actuales profesores universitarios. 
Fuente: ANECA (http://www.aneca.es/Programas-de-evaluacion/ACADEMIA)

Los méritos para obtener una calificación B para la categoría de titular de Universidad se regulan para cada comisión en relación a dos apartados: los méritos obligatorios, y los méritos complementarios (que no son de obligada presentación, pero que pueden compensar deficiencias en los méritos obligatorios). La comisión D18 corresponde a Ciencias Sociales, y según se indica, los méritos presentados en este apartado por los solicitantes deberán responder a una trayectoria investigadora coherente, independiente y activa en los últimos años, avalada por la vinculación a proyectos competitivos (ANECA, 2016).

En el apartado de contribuciones científicas publicadas, se indica que deben ser mínimo 20 publicaciones, de las cuales, al menos, siete deberán ser artículos de revista, en las que al menos, un tercio de las publicaciones aportadas el solicitante deberá firmar como primer autor, y se deberán aportar un mínimo de cuatro artículos en revistas del nivel 1 y 2.

Para su evaluación se considerarán cuatro niveles de revistas: nivel 1 (indexadas en los Q1 y Q2 del Journal Citation Reports, JCR y Ql Scimago Journal Rank, SJR), nivel 2 (incluidas en el Q3 JCR y Q2 SJR), nivel 3 (incluidas en el Q4 JCR y Q3 SJR, con sello FECYT o en Emerging SCI) y nivel 4 (incluidas en el Q4 de SJR, otras clasificaciones o sin factor de impacto).

La obligación de publicar en revistas indexadas en el Journal Citation Reports (JCR), y en especial la exigencia de publicar en revistas incluidas en el primer y segundo cuartil en el nivel 1 , y el tercer cuartil para el nivel 2, implica que el reconocimiento oficial de la calidad investigadora lo otorga el factor de impacto que determina una empresa privada, Thomson Reuter, con sede en Estados Unidos, aspecto sobre el que volveremos más adelante-. Pero, además, supone tener en cuenta las características concretas de este índice: la edición del Journal Citation Reports (JCR), con datos de 2015, comprende 11.962 revistas, de las cuales 8.757 corresponde a la edición de Ciencias (Science JCR) y 3.201 revistas a Ciencias Sociales (Social Sciences JCR). En relación a España, aparecen indizadas 119 revistas, 72 en la edición de Ciencias y 47 en la edición de Ciencias Sociales (Bibliotecas USAL, 2015). En la edición de Ciencias Sociales de 2014 había una más, 48, y en ella se incluían diferentes disciplinas: en Derecho constan 2 revistas, en Economía 8, en Educación 9, en Humanidades 17, en Políticas y Sociología 6, y multidisplinares otras 6. En el área de Políticas y Sociología las revistas incluidas son Comunicar, Historia y Política, Revista Española de Investigaciones Sociológicas, Revista de Estudios Políticos, Revista Internacional de Sociología, y Revista de Psicología Social (Biblioteca UNED, 2014). 
Tabla 2. Acreditación profesor titular de Universidad: Programa ACADEMIA 2.0 y 3.0

\begin{tabular}{|c|c|c|c|}
\hline $\begin{array}{c}\text { RAMA } \\
\text { CIENCIAS } \\
\text { SOCIALES }\end{array}$ & ACADEMIA 2.0(Versión 2008) & ACADEMIA2.0 (Versión 2011) & $\begin{array}{l}\text { ACADEMIA } 3.0 \text { (2015)NIVEL B } \\
\text { (BUENO/SUFICIENTE) }\end{array}$ \\
\hline Investigación & $\begin{array}{l}\text { La publicación de un mínimo de } 8 \\
\text { artículos en revistas recogidas en los } \\
\text { índices de calidad, de las cuales un } \\
\text { porcentaje significativo deben corres- } \\
\text { ponder a revistas indexadas con un } \\
\text { índice de calidad relativa. }\end{array}$ & $\begin{array}{l}\text { Un mínimo de } 8 \text { artículos en revistas recogidas } \\
\text { en los índices que se mencionan en el anexo I } \\
\text { "Indicios de calidad", de las cuales un porcenta- } \\
\text { je significativo deben corresponder a revistas } \\
\text { indexadas del JCR. }\end{array}$ & $\begin{array}{l}\text { Un mínimo de } 20 \text { publicaciones, de las cua- } \\
\text { les: } \\
\text { - Al menos siete deberán ser artículos de } \\
\text { revista. } \\
\text { - Se deberán aportar un mínimo de cuatro } \\
\text { artículos en revistas del nivel } 1 \text { y } 2 \text {. }\end{array}$ \\
\hline
\end{tabular}

Fuente: Elaboración propia a partir de ANECA (http://www.aneca.es/Programas-de-evaluacion/ACADEMIA). 
En relación al índice de 2016, y teniendo en cuenta el criterio de la posición en diferentes cuartiles que exige ANECA, en el área de Ciencias Sociales sólo aparece una revista en el primer cuartil, la revista Comunicar, y ninguna en el segundo cuartil. Por su parte, de un total de 85 revistas de sociología recogidas por la Federación Española de Sociología, las únicas dos revistas específicas de Sociología que aparecen en el JCR son La Revista Española de Investigaciones Sociológicas y la Revista Internacional de Sociología, ambas en el $4^{\circ}$ cuartil. Según la clasificación ANECA de niveles de revistas para su evaluación, estas revistas están incluidas en el nivel 3, y por tanto, cualquier artículo publicado en ellas no podría contribuir a cumplir el requisito mínimo de alguno/s de los cuatro artículos publicados en revistas del nivel 1 y 2.

Este dato guarda una estrecha relación con la proporción de revistas en los diferentes cuartiles y disciplinas. Si tenemos en cuenta el listado de 2016, en el que se incluyen revistas de 81 países, con 11.365 revistas, de 234 disciplinas, se observa que en el área de Sociología solo 71 revistas de todo el mundo ocupan el puesto Q1 o Q2, de las cuales no hay ninguna en lengua castellana (BUS, 2016).

Por su parte, en la categoría Sociology and Political Science 2015 del SCImago Journal Rank (SJR), de las 21 revistas españolas que aparecen, ninguna está en el Q2, cuartil necesario para cumplir el requisito nivel 2 de ANECA. Si consideramos las 160 revistas que aparecen en la categoría Ciencias Sociales, entre las que se encuentran en el Q1 y Q2 están Comunicar (Q1), Profesional de la Información (Q2), Comunicación y Sociedad (Q2), Revista Latina de Comunicación Social (Q2), entre otras, pero ninguna de las que recoge la Federación Española de Sociología.

\section{Journal Citation Report (JCR) y SCImago Journal Rank (SJR)}

El indicador más valorado por los organismos de evaluación de la actividad investigadora es el Journal Citation Report (JCR), que mide el impacto de una revista en función del número de veces que se cita por término medio un artículo publicado en una determinada revista recogida en la Web of Science (WOS). Esta base de datos proporciona indicadores de la producción científica por revistas, entre otros el Factor de Impacto (FI), y cuenta con dos series o ediciones: Science y Social Science. Se consulta en la plataforma ISI Web of Knowledge (WOK) y el responsable es la empresa Thomson Reuters, que ha heredado la tradición del antiguo Institut of Scientific Information de Philadelphia (ISI), dirigido por Eugene Garfield (BUS, 2015).

Asumiendo el vínculo y la relevancia que las revistas científicas han tenido, y siguen teniendo en relación a los sistemas de I+D (Fernández Esquinas, 2016; Torres Albero, 2016), lo que interesa señalar aquí son precisamente las consecuencias negativas que se derivan de la utilización por parte de las revistas científicas de un criterio absolutamente mercantilizador (la bibliometría). En este sentido, el Journal Citation Report (JCR) ha recibido muchas críticas. En función de los datos expuestos anteriormente hay dos que destacan de forma notable: por un lado, la renuncia al propio idioma ante la exigencia de publicar en inglés, por otro, la inclinación por las ciencias físico-naturales y tecnológicas en perjuicio de las ciencias sociales que 
domina en el JCR. El hecho de que no haya ninguna revista de todo el mundo en lengua castellana en Sociología en el Q1 y Q2 del JCR, de un total de 71, es un síntoma claro de lo que se ha denominado como colonialismo de la investigación: es decir, renunciar, en primer lugar, a la lengua castellana, y en segundo lugar tener que orientar la investigación hacia temas con interés internacional. Es evidente que este índice favorece a publicaciones y autores anglosajones, a la vez que introduce un claro sesgo relacionado con el idioma y la geografía que margina a las revistas que no publican en inglés.

Además, las ciencias sociales salen perjudicadas al ser evaluadas con un sistema que fue generado para las ciencias físico-naturales. Este sistema no tiene en cuenta algunas diferencias epistemológicas entre las ciencias físico-naturales y las ciencias sociales como, por ejemplo, el hecho de que el objeto de estudio en las ciencias sociales suele ser menos universalista y más local, por lo que su difusión e impacto suelen tener límites nacionales. Otra de las características es que existe una mayor variedad de formatos para difundir el conocimiento, además del paper (Reig, 2014). Sin embargo, y debido a las pautas de estandarización y convergencia internacional adoptadas por las agencias evaluadoras, se ha producido una traslación de determinadas prácticas de difusión de la ciencia (los papers), asociadas en origen a las ciencias físico-naturales, al campo de las ciencias sociales (más asentadas en otras formas de difusión, que incluyen no sólo la publicación de artículos, sino de monografías, libros, etc.).

Además, existen otro tipo de limitaciones relacionadas con el factor de impacto, independientemente del área de conocimiento que se esté evaluando. Una de ellas, se relaciona con que algunos artículos pueden recibir citas años después de haber sido publicados, hecho frecuente en algunas disciplinas, por lo que, aunque tienen impacto, no se mide 7 . Otras limitaciones y críticas se vinculan con el hecho de que algunos editores animan a los autores a citar superfluamente artículos de la misma revista en la cual publican, o se realizan acuerdos entre revistas para citarse mutuamente. Situaciones que, de ser detectadas, implica la eliminación por un tiempo del JCR, pero que sin embargo se producen. Por otro lado, también es conocida la profusión de citas espurias: aquellas que se incluyen por puro protocolo. En este sentido, Simkim \& Roychwdhury (2007) estimaron, por ejemplo, que teniendo en cuenta la repetición de erratas (es decir, las citas que se copian sin más de las referencias bibliográficas) sólo un $23 \%$ de los artículos que se citaban habrían sido leídos por los autores de los artículos. Por último, no hay que olvidar que el número de citas no es un equivalente de la calidad del trabajo que se cita. En muchas ocasiones, tan sólo se citan artículos para ser criticados o valorados negativamente.

La otra base de datos bibliográfica que ANECA admite para otorgar el nivel 2 a los artículos es Scopus, propiedad de la empresa Elsevier. La base de datos Scopus incluye resúmenes y citas de literatura revisada por pares, y abarca más de 60 millones de documentos publicados en más de 21.500 revistas, series de libros y actas de congresos de aproximadamente 5.000 editores. Scopus también cubre todos los campos de investigación más importantes, con 6.900 títulos en Ciencias físicas, 6.400 en Ciencias de la salud, 4.150 en Ciencias de la vida y 6.800 en Ciencias

7 El cálculo, por ejemplo, del F.I. 2013 de una revista $=n^{\circ}$ de citas que han recibido en 2013 los artículos publicados en 2011 y 2012 / nº de art. publicado en los años 2011-2012 
sociales (esta última, incluye unos 4.000 títulos relacionados con las Artes y humanidades) (FECYT, 2016).

El portal de evaluación de la base de Datos Scopus es SCImago Journal \& Country Rank. La plataforma debe su nombre al SCImago Journal Rank (SJR), que evalúa las revistas españolas con Factor de impacto SJR. Scimago Journal Rank ha sido desarrollado por SCImago, grupo de investigación del Consejo Superior de Investigaciones Científicas (CSIC), y de las universidades de Granada, Extremadura, Carlos III (Madrid) y Alcalá de Henares, que desarrolló su métrica científica (BUS, 2015).

\section{Más allá de la evaluación del profesorado}

El hecho de que ANECA y CNEAI hayan apostado por la publicación de papers como criterio de evaluación preferente, en detrimento de cualquier otro tipo de producción y difusión científica, y que estos textos tengan que estar publicados por revistas científicas indexadas principalmente en dos empresas privadas (Thomson Reuters -WoS- y Elsevier - Scopus-), en menoscabo de otros formatos, bases de datos y vías de expansión de la investigación, lleva a la cuestión de la mercantilización del conocimiento científico a través, en este caso, de determinadas revistas consideradas como de alto impacto. Lo que, cuanto menos, cuestiona el principio de libertad de publicación. Por ello, más allá de las implicaciones que tiene la utilización del JCR en la evaluación de profesorado, la cuestión que trasciende es que el conocimiento ha entrado desde hace años en el circuito del mercado ${ }^{8}$.

Este aspecto tiene diferentes implicaciones. Una de ellas se relaciona con el hecho de que los resultados de la labor que realizan los investigadores, en base a los proyectos pagados con dinero público, se publican en revistas de acceso privado. El otro, que para acceder a esas revistas hay que pagar un canon que revierte a una corporación privada. De esa manera, el conocimiento, que constituye en su origen un bien común y un servicio que procede y tiene que revertir en la sociedad, acaba de este modo privatizado.

La decisión por parte de ANECA y CNEAI de optar por utilizar los índices de impacto del JCR, como indicador de calidad para la evaluación del profesorado y de los tramos investigadores para la concesión de sexenios de investigación, ha supuesto por otra parte relegar indicadores de calidad desarrollados para las revistas científicas españolas de Ciencias Sociales y Humanidades como DICE (Difusión y Calidad Editorial de las Revistas Españolas de Humanidades y Ciencias Sociales y Jurídicas) y RESH (Revistas Españolas de Ciencias Sociales y Humanidades).

Según recoge Giménez Toledo (2015), el sistema DICE fue desarrollado por parte del CSIC (Consejo Superior de Investigaciones Científicas), y al ser una institución pública, no recibía dinero por parte de las revistas que estudiaba y catalogaba, ni tampoco directamente de los investigadores/profesores, etc., que la consultaban (Díez Gutiérrez, 2016). Otros proyectos públicos de prestigio que se

8 En 2012 tan solo 5 grandes empresas de la información científica, técnica y médica, acaparaban el 70\% del mercado (Baiget, T.y Olea, I. 2014). 
desarrollaron fueron RESH, (Revistas Españolas de Ciencias Sociales y Humanidades) como sistema de información que integra indicadores de calidad para las revistas científicas españolas de Ciencias Sociales y Humanidades financiado por el Plan Nacional de I+D+i en 2007. También hay que destacar INRECS (Índice de impacto de las revistas españolas de Ciencias Sociales), así como INRECJ e INRECH, para ciencias jurídicas y humanidades respectivamente, por parte de la Universidad de Granada. Por último, el aún vigente MIAR (De Mata et al., 2013), y la creación de iniciativas desde el ámbito más político/institucional, como el sello de calidad FECYT (Fundación Española para la Ciencia y la Tecnología).

Los recortes presupuestarios han supuesto la paralización de DICE en 2013, y la de INRECS, INRECJ e INRECH de la Universidad de Granada en el año 2014. Sin embargo, y según el análisis que realizan Tomás Baiget e Isabel Olea, "España a través de FECYT, adquirió en 2008 la base de datos cerrada 1900-1944 Sciencie Citation Index Expanded por 2.320.000 dólares USA (1.488.985,24 euros). En 2013 abonó 3.136.000€ por el acceso anual a la WoS y Scopus $(1.902 .258 €$ y $1.233 .742 €$ respectivamente). Y recuperó 2.063.641€ pagados por las universidades y demás centros de investigación. Es decir, FECYT subvenciona el acceso a ambas bases de datos con 1.072.359 €” (Baiget y Olea, 2014, p. 251).

El hecho de que los sistemas nacionales de evaluación desaparezcan no solo tiene implicaciones para la evaluación del profesorado. Supone también que muchas revistas que se editan desde las universidades españolas, con fondos públicos que reciben para tal fin, son minusvaloradas en los procesos de evaluación que tienen en cuenta exclusivamente la WoS (Giménez-Toledo, 2015).

Para llegar a esta situación es necesario, no obstante, la cooperación de las administraciones públicas (en financiación y difusión) (Díez Gutiérrez, 2016), y de esta forma, lo que comenzó siendo en su origen un sistema de evaluación de las publicaciones científicas, se convierte en un sistema más complejo, en el que están en "el epicentro (la empresa Thomson Reuters), las Administraciones públicas de Ciencia e Innovación como participantes necesarios, índices de citas e informes de factor de impacto (JCR), los evaluadores externos y los investigadores y consumidores de estas producciones. Además, habría que incluir normativas oficiales, agencias de evaluación, otras bases o recursos para la valoración, otras revistas con índices de calidad relativos, etc.” (Herrán, 2012, p. 4).

La situación que se genera se asemeja, según la metáfora que utiliza Herrán, a la ciudad medieval agolpada alrededor del castillo en cuyo interior vive una élite que publica en revistas JCR, y extramuros se agolpa el campesinado, es decir, los docentes/investigadores que día a día contribuyen al enriquecimiento de la ciencia con su trabajo, pero sin tanto reconocimiento. Y lo que es peor, "como consecuencia de esta ciudad medieval, algunos autores no enviarán artículos a revistas peor situadas en las bases de datos, ni aun bajo invitación (...) porque hacerlo "no les valdrá para nada” (Herrán, 2012: 14). Por su parte, Herzog et al. (2015), utilizan la figura del panóptico de Foucault para dar cuenta de la relación que se genera entre la observación, (en forma de índices bibliométricos), poder (en forma de distribución de recursos), y comportamientos individuales (Herzog et al. 2015 p. 78). La cuestión que subyace tiene que ver, por tanto, con quién decide sobre las estructuras y los criterios de evaluación y el tipo de subjetividades que están generando. 
Por otro lado, algunas de las implicaciones de este cambio se relacionan también con la limitación en el acceso a la carrera docente y la precarización del profesorado universitario, ante lo que también hay que preguntarse qué defensa tienen los investigadores/profesores frente a las estructuras y dictámenes de evaluación.

Según el informe la Universidad española en Cifras, 2014-2015 publicado por la Conferencia de Rectores de las Universidades Españolas (CRUE), la crisis económica y el descenso en la financiación pública de las Universidades han tenido un efecto notable en la reducción y el envejecimiento de las plantillas universitarias. Así, entre 2010 y 2014, se han destruido 3.486 puestos de profesores docentes e investigadores (PDI). Por su parte, la aplicación de la tasa de reposición a partir de 2012 ha reducido, por la vía de la jubilación, la presencia de catedráticos y titulares de universidad y ha impedido "la promoción de más de 5.000 profesores acreditados para el acceso a estas categorías profesionales. El resultado es que el perfil profesional que a finales del año 2014 presentan las plantillas universitarias de profesorado es, tanto cuantitativa como cualitativamente, muy similar al que ofrecían a finales del año 2006” (CRUE, 2016, p.75). Teniendo en cuenta estos datos sobre el cuello de botella que ha generado que más de 5.000 profesores acreditados no hayan podido acceder a las categorías de titular o catedrático, no faltan voces que argumentan que el endurecimiento de los criterios para la acreditación puede ser también una táctica que ayude a limitar el número de acreditaciones, y consecuentemente, la demanda de plazas para esas categorías.

Además, desde la creación del Espacio Europeo de Educación Superior, principalmente, y como respuesta a las transformaciones que se están produciendo en la universidad española, cada vez son más numerosas las aportaciones que ponen de manifiesto la progresiva consolidación de políticas de carácter neoliberal. A partir del Plan Bolonia, en la Universidad se han introducido los sistemas de contratación de la empresa privada (contratado doctor, ayudante doctor, asociado), que han ido desplegando un escenario que busca incentivar ese tipo de productividad, al tiempo que ha generado un aumento de la segmentación y precarización del colectivo docente (Llopis, Sevilla, Marugán y Cruces, 2012).

Ante el malestar que se ha generado, están consolidándose colectivos, como Indocentia, de la Universidad de Valencia. Algunas de las cuestiones sobre las que centran su trabajo giran en torno a analizar en qué se convierte la investigación sometida a criterios y rankings que valoran principalmente lo cuantificable, exhibible y comercializable, o en qué se convierte el ejercicio de la docencia cuando se considera una actividad de segunda (Fernández-Savater, 2016).

En otros casos la reflexión y el debate se ha realizado desde posiciones individuales a través de artículos científicos (como los que han servido de referencia para este artículo y se incluyen en la bibliografía), o desde los comités de redacción de revistas científicas, como es el caso del número 32 de Arxius de Ciències Socials, en 2015.

Otras veces se ha optado por realizar medidas judiciales ${ }^{9}$, porque la precarización de las condiciones laborales del profesorado es un panorama frecuente en los

9 En el transcurso del cierre de este artículo la prensa publicaba una información en la que informaba de que la Plataforma de Profesores Asociados de la CGT había llevado a juicio a la Universitat de Barcelona por las condiciones laborales de los profesores asociados. Disponible en: http://www.eldiario.es/catalunya/educacion/UB-juicio-contratos-profesores-asociados 0 6 629937979.html 
centros universitarios públicos españoles, y algunos han iniciado ya la batalla desde extramuros, como los profesores asociados de la Universidad de Valencia que han denunciado al Parlamento Europeo sus condiciones laborales.

\section{Cierre}

Además de las dificultades que la bibliometría tiene a la hora de evaluar las contribuciones sustantivas en el avance de la ciencia, así como para valorar el impacto real de las mismas en relación con los problemas concretos de la sociedad, o para orientar la I+D (Fernández Esquinas, 2016), ese afán por el recuento descontextualizado de artículos y citas, antes que excelencia, lo que está generando es un entramado de "habilidades", por parte de algunos científicos y docentes, en aras de conseguir la publicación de sus papers en las revistas mejor posicionadas a cambio de abandonar determinadas áreas temáticas y/o metodologías de investigación (menos publicables).

Además, se está desatendiendo la producción de monografías o libros en los que cabe una reflexión más pausada y prolija de los temas investigados durante años; se tiende al abandono de temas locales en beneficio de temas globales (con mejor pronóstico para su publicación en revistas internacionales); y en consecuencia, se lucha por publicar en revistas internacionales habida cuenta de las restricciones de algunas empresas (léase Thomson Reuters) al mercado editorial no anglosajón o se tiende a la búsqueda de resultados positivos en las investigaciones (debido a que los negativos tienden a ser publicados en menor medida).

Por último, entre los efectos "no previstos" de todo este sistema evaluador conviene resaltar la creación tácita de redes de citas para el apoyo mutuo de determinados grupos de investigación o editoriales; y, por supuesto, las guerras de citas frente a posibles competidores, etc. De tal manera que la pretendida libertad de la Ciencia, así como la difusión y publicación del conocimiento como objeto de la misma, se van desvaneciendo como un azucarillo en el agua ${ }^{10}$.

En definitiva, y como bien señaló Bourdieu, en este sistema de reconocimiento de la ciencia, los científicos tratan de comportarse como inversores que intentan maximizar sus inversiones tratando de seleccionar, qué investigan, con quién se alían, a quién citan, etc. Todo ello con el objetivo de obtener la máxima recompensa: la publicación en las revistas de mayor impacto (Torres Albero, 2016).

Por otro lado, el hecho de que el CNEAI haya optado, también, por utilizar los índices de impacto (bibliometría) como indicador de calidad para la evaluación del profesorado y los tramos investigadores y la concesión de sexenios de investigación, ha derivado hacia una situación en la que, los sexenios, que habían sido planteados en origen como un complemento retributivo para la incentivación investigadora (un incentivo), han terminado por convertirse en un criterio de de-

10 Señala A. Embid (2017) que donde mejor se ha expresado todo esto es en la República Federal Alemana, donde a partir del art. 5.3 de su Constitución que dice: "El arte y la ciencia, la investigación y la enseñanza son libres”, se ha llevado a cabo un profundo debate político, social y jurídico, con varias sentencias del Tribunal Constitucional federal. 
marcación en perjuicio de la docencia. Transformando a ésta en una carga (“carga docente”) para aquellos que no consigan sexenios.

Conseguir el máximo número de artículos en revistas indexadas en las bases de datos citadas, supone el gran reto para lograr no solo un plus económico, sino sobre todo para lograr prestigio en la escala académica y descarga de horas de docencia. Por otra parte, se trata de adecuarse a los criterios del CNEAI, es decir, realizar aquella labor investigadora que mejor encaje con ellos. De esa manera, se estimula un sistema de productividad que cada vez se aleja más de los principios de libertad, universalismo, compromiso social, que deberían regir el espíritu y la vida de la comunidad científica.

Con este telón de fondo, todo parece indicar que ante la falta de una política científica fuerte que oriente líneas de investigación, lidere la creación y desarrollo de centros y equipos de investigación y dote de recursos humanos y económicos suficientes, las agencias de evaluación han introducido determinados principios que van a regir no solo la forma de producir conocimiento, sino el desarrollo, freno o estancamiento de las carreras académicas. Convirtiéndose de ese modo en ejes vertebradores del sistema nacional de investigación (Fernández Esquinas et al., 2016). Si bien es cierto que estas agencias han ayudado al desarrollo del sistema de la ciencia en España, no lo es menos que han generado consecuencias negativas, que no solo tienen que ver con las tensiones dentro del mundo académico (carreras académicas, estratificación, reparto entre docencia e investigación, etc.), sino que cuestionan la misma relación con el principio de libertad, como característica básica que supuestamente ha de regir el mundo de la ciencia.

\section{Referencias bibliográficas}

ANECA (2016). Programa ACADEMIA de acreditación nacional para el acceso a los cuerpos docentes universitarios. Disponible en: http://www.mecd.gob.es/servicios-alciudadano-mecd/catalogo/general/educacion/academia/ficha/academia.html

Baiget, T., Olea, I. (2014). "Econonomía, industria y mercado de la información en 2013”, en Guallar, J. (dir.) Anuario ThinkEPI 2014, Análisis de Tendencias en información y documentación, Editorial UOC, 243-255.

Biblioteca UNED (2014). Revistas españolas en JCR edición 2014. Disponible en: http://www2.uned.es/biblioteca/guia_impacto/gf2.

Biblioteca USAL (Universidad de Salamanca) (2015). Actualización JCR (Journal Citation Reports) 2015. Disponible en: https://bibliotecas.usal.es/noticia/actualizacion-jcrjournal-citation-reports-2015

Bourdieu, Pierre (2003/2001). El oficio de científico. Ciencia de la ciencia y reflexividad. Barcelona, Anagrama.

BUS (Biblioteca Universidad de Sevilla) (2015). "Factor de impacto". Guía de la BUS. Investigación. Disponible en: http://guiasbus.us.es/factordeimpacto

BUS (Biblioteca Universidad de Sevilla) (2016): JCR Social Sciences ( $1^{\circ}$ y $2^{\circ}$ cuartil). Disponible en: http://bib.us.es/sites/bib3.us.es/files/jcr_social_2015.pdf

Cabrera, P., Nogués L., y García Giráldez, T. (2013). El papel de las revistas de Trabajo Social en el desarrollo de la disciplina. XII Congreso Estatal de Trabajo Social, Marbella 14-16 noviembre.

CRUE (2016). La Universidad Española en Cifras, 2014/2015. CRUE. 
De Azcárraga Feliu, J. A. (2014). Por qué debería cambiar la selección del profesorado universitario. Nueva Revista de Política, cultura y Arte, (151), 52-64

Díez Gutiérrez, E.J. (2016, 5 de octubre). Reino de España: Más allá del negocio de los sexenios universitarios. Nuevatribuna.es.

Disponible en: http://www.nuevatribuna.es/articulo/sociedad/mas-alla-negociosexenios-universitarios/20161005102328132368.html

Embid, A. (2017). Investigación: la indefinición del modelo organizativo español en la actualidad. Universidad. El blog de Studia XXI.

Disponible en: http://www.universidadsi.es/investigacion-la-indefinicion-del-modeloorganizativo-espanol-la-actualidad/

FECYT (Fundación Española para la Ciencia y la Tecnología) (2016). Indicadores bibliométricos de la actividad científica española 2005-2014. Edición 2016. Apéndice A. Metodología y Fuentes de datos. Disponible en: (http://www.buc.unican.es/sites/default/files/DOCS/guia_herramientas_de_evaluacion 2013.pdf

Fernández Esquinas et al. (2009). Anillos de crecimiento en el árbol de la ciencia. La evolución institucional del Consejo Superior de Investigaciones Científicas. Revista Internacional de Sociología, 67 (2), 251-284.

Fernández Esquinas et al. (2016). Las revistas de ciencias sociales en los sistemas de I+D. Notas sobre política editorial para revistas de sociología. RES, Vol. 25 (3), 427-442.

Fernández-Savater, A. (2016, 2 de febrero). Disciplinar la investigación, devaluar la docencia: cuando la Universidad se vuelve empresa. El diario.es. Disponible en: http://www.eldiario.es/interferencias/Disciplinar-investigacion-devaluar-docenciaUniversidad 6 486161402.html

Fernández-Zubieta, A. (2014). ERAWATCH Country Reports 2013: Spain (9279345265). Disponible en: https://ec.europa.eu/jrc/sites/default/files/formcr2013_es_final.pdf

Giménez-Toledo, E. (2015). La evaluación de la producción científica: breve análisis crítico. Relieve, 21(1), 1-9. DOI: 10.7203/relieve.21.1.5160

Herrán, A. (2012). Algunas críticas a la evaluación del profesorado universitario centrada en el impacto. En A. de la Herrán y J. Paredes (Coords.), Promover el cambio pedagógico en la universidad. Madrid: Pirámide (Colección 'Pedagogía y Didáctica').

Herzog, B., Pecourt, J., y Hernàndez i Dobon, F. J. (2015). La dialéctica de la excelencia académica: de la evaluación a la medición de la actividad científica. Arxius de Ciencias Sociales, 32, 69-82.

Llopis, E.S., Sevilla, C. Marugán y Cruces, J. (2012). Universidad: ¿Hacia un cambio de modelo? Informe de la Fundación $1^{\circ}$ de Mayo, 49, Julio 2012. Disponible en: http://firgoa.usc.es/drupal/node/50922.

De Mata, J., Gandarillas, R., Martinez, J. (2013) Guía de herramientas para evaluar la investigación, Vicerrectorado de Calidad e Innovación Educativa, Biblioteca Universitaria, Universidad de Cantabria.

Reig, Ramón, (2014) La investigación dependiente: crítica estructural al sistema JCR Ámbitos, Revista Andaluza de Comunicación, 27.

Simkin M.V. \& Roychowdhury, V. P. (2006). Do you sincerely want to be cited? Or: read before you cite. Significance Sept. 179-181.

Torres Albero, C. (2016). Los tres primeros minutos de la Revista Española de Sociología (RES). RES, Vol. 25 (3), 445-451. 